\title{
AUTONOMOUS INTELLIGENT FLIGHT CONTROL OF FIXED-WING UAV BASED ON ADAPTIVE NEURO-FUZZY INFERENCE SYSTEM
}

\author{
Amr Sarhan ${ }^{1}$, Shiyin Qin ${ }^{2}$ \\ ${ }^{1}$ School of Automation Science and Electrical Engineering, Beihang University, China \\ ${ }^{2}$ School of Automation Science and Electrical Engineering, Beihang University, China
}

\begin{abstract}
This paper develops an autonomous flight controller for fixed-wing UAV based on ANFIS. Three ANFIS modules are designed for controlling the altitude, the heading angle, and the speed of the UAV. In this way, the UAV position is controlled in threedimensional space: altitude, longitude, and latitude position. The simulation results show the capability of the designed approach and its very satisfied performance with good stability and robustness against UAV parametric uncertainties and external wind disturbance.
\end{abstract}

Keywords: UAV, autonomous flight control, ANFIS

\section{INTRODUCTION}

Nowadays, UAVs are becoming more useful due to their critical missions in civil and military applications. For military applications, UAVs can be used for surveillance, target designation, countermeasures, weapon delivery, etc. For civil applications, UAVs can perform forest fire detection, pollution detection, weather detection, agriculture, etc [1]. Many significant characteristics make UAVs very important such as small size, inexpensive, and their ability to do complex maneuvers due to the lack of a human pilot $[2,3]$. Besides, advancements in aerodynamics, propulsion, computers, and sensor technologies allowed UAV to have capabilities that were not available before [4].

The key aspect of UAV design is the flight control system [5]. The traditional flight control system approach is PID algorithm due to its simplicity, easy for implementation in hardware and software, and does not require maintenance [6]. However, the resulting flight controller usually has lower adaptability and does not produce a good performance when tested in more realistic UAV environment [7,8]. The traditional approach for enhancing the PID performance is to use PID with gain scheduling [9]. However, the switching between different controllers sometimes is roughness and it is necessary to design a single flight controller to operate for a certain flight envelope [10,11]. Different control techniques have been developed and verified successfully for UAV's flight control systems such as optimal control, predictive control, adaptive control, and robust control $[12,13]$.

In recent years, various nonlinear flight control algorithms have been utilized in small UAV flight control systems such as backstepping [14], Lyapunov function [15], and nonlinear dynamic inversion [16]. Notwithstanding backstepping and Lyapunov algorithms are too complex to design. In addition, the nonlinear dynamic inversion algorithm strongly depends on precise nonlinear models which limit the performance when applied to UAV approximate nonlinear models [17].
On the other side, the fuzzy logic approach has the ability to compensate the effects of nonlinearity and uncertainties, also has strong robustness independent of a mathematical model of the system. In addition, designing fuzzy logic controller is fast, simple, and easily maintained because the rules can be understood and explained linguistically by the human experts [18].

Furthermore, the neural network controller is suitable for uncertain, dynamic, and non-linear systems [19]. It helps in eliminating or reducing the necessity for the offline gain tuning. The neural network controller is featured by online adaptability. This can be utilized in designing real-time control laws in order to overcome the effects of uncertainties and nonlinearity in system dynamics. Moreover, designing an adaptive flight controller for UAV based on the neural network will decrease the effort required for modeling the flight systems and identified the flight platform. By combining fuzzy logic and neural network controller, more strong and versatile models called "adaptive neuro-fuzzy inference system (ANFIS)" has been developed.

ANFIS is better than the neural network with a one-hiddenlayer in the architecture, especially when dealing with parametric uncertainties [20]. It has excellent fault-tolerance characteristic, which can permit the UAV parameters to vary in practical flight situation [21]. ANFIS controller has advantages of solving the control problem of uncertain systems by means of a fuzzy system and optimizing controller parameters through the self-learning ability of a neural network [22]. In this paper, an autonomous flight controller for fixed-wing UAV is designed by utilizing an adaptive neuro-fuzzy inference system (ANFIS). Three fuzzy modules are designed to achieve this objective. Two modules are designed for the longitudinal autopilot to achieve the desired altitude and airspeed. The other module is designed for the lateral autopilot to achieve the desired heading angle. In this way, the UAV position is controlled in the three-dimensional space. 


\section{UAV NONLINEAR DYNAMIC MODEL}

The dynamics of the UAV in motion can be given by Newton's $2^{\text {nd }}$ law which is suitable in the inertial frame. A complete 6-DOF nonlinear Aerosonde UAV dynamic model (with body fixed frame) [23] is demonstrated in the forthcoming discussion. Aerosonde UAV is utilized as a test air vehicle because of the availability of the specifications and the flight parameters. The Aerosonde UAV is fixedwing UAV with 6-DOF [24], its nonlinear mathematical model is illustrated by the following 12 dynamic variables: body frame velocities $(u, v, w)$, Euler angles $(\varphi, \theta, \psi)$, angular velocities $(p, q, r)$, and inertial positions $\left(p_{N}, p_{E}, h\right)$, on the other hand, the model depends on external forces $\left(f_{x}, f_{y}, f_{z}\right)$ and moments $(l, m, n)$. The dynamic model is summarized in (1)-(4).

$$
\left\{\begin{array}{l}
\dot{u}=r v-q w+\frac{f_{x}}{m} \\
\dot{v}=p w-r u+\frac{f_{y}}{m} \\
\dot{w}=q u-p v+\frac{f_{z}}{m}
\end{array}\right.
$$

$\left\{\begin{array}{c}\dot{\varphi}=\mathrm{p}+\mathrm{q} \sin \varphi \tan \theta+\mathrm{r} \cos \varphi \tan \theta \\ \dot{\theta}=\mathrm{q} \cos \varphi-\mathrm{r} \sin \varphi \\ \dot{\psi}=\frac{\mathrm{q} \sin \varphi+\mathrm{r} \cos \varphi}{\cos \theta}\end{array}\right.$

$\left\{\begin{array}{l}\dot{\mathrm{p}}=\mathrm{c}_{1} \mathrm{qr}+\mathrm{c}_{2} \mathrm{pq}+\mathrm{c}_{3} \mathrm{l}+\mathrm{c}_{4} \mathrm{n} \\ \dot{\mathrm{q}}=\mathrm{c}_{5} \mathrm{pr}-\mathrm{c}_{6}\left(\mathrm{p}^{2}-\mathrm{r}^{2}\right)+\mathrm{c}_{7} \mathrm{~m} \\ \dot{\mathrm{r}}=\mathrm{c}_{8} \mathrm{pq}-\mathrm{c}_{2} \mathrm{qr}+\mathrm{c}_{4} \mathrm{l}+\mathrm{c}_{9} \mathrm{n}\end{array}\right.$

$$
\left\{\begin{array}{c}
\dot{\mathrm{p}}_{\mathrm{N}}=\mathrm{u} \cos \theta \cos \psi+\mathrm{v}(\sin \varphi \sin \theta \cos \psi-\cos \varphi \sin \psi) \\
+\mathrm{w}(\sin \varphi \sin \psi+\cos \varphi \sin \theta \cos \psi) \\
\dot{\mathrm{p}}_{\mathrm{E}}=\mathrm{u} \cos \theta \sin \psi+\mathrm{v}(\cos \varphi \cos \psi+\sin \varphi \sin \theta \sin \psi) \\
+\mathrm{w}(\cos \varphi \sin \theta \sin \psi-\sin \varphi \cos \psi) \\
\dot{\mathrm{h}}=\mathrm{u} \sin \theta-\mathrm{v} \sin \varphi \cos \theta-\mathrm{w} \cos \varphi \cos \theta
\end{array}\right.
$$

where $c_{1}, \ldots, c_{9}$ are functions of moments of inertia $\left(J_{x}, J_{y}, J_{z}\right)$. The forces $\left(f_{x}, f_{y}, f_{z}\right)$ and moments $(l, m, n)$ that acts on the UAV are mainly due to three sources: gravity, aerodynamics, and propulsion. These variables depend on UAV mass (m), gravity (g), Euler angles, density of air $(\rho)$, airspeed $\left(\mathrm{V}_{\mathrm{a}}=\sqrt{\mathrm{u}^{2}+\mathrm{v}^{2}+\mathrm{w}^{2}}\right)$, surface area of the wing $(S)$, angular velocities, angle of attack $\left(\alpha=\tan ^{-1}\left(\frac{w}{u}\right)\right)$, side slip angle $\left(\beta=\sin ^{-1}\left(\frac{\mathrm{v}}{v_{\mathrm{a}}}\right)\right)$, control surface configuration (ailerons $\delta_{\mathrm{a}}$, elevator $\delta_{\mathrm{e}}$, rudder $\delta_{\mathrm{r}}$ ), engine acceleration $\left(\delta_{t}\right)$, area, aerodynamic coefficient and torque of the propeller, the efficiency of the engine, and the aerodynamic coefficients (see [25] for details).

The three basic variables for controlling the UAV are longitudinal speed $\mathrm{u}(\mathrm{t})$, altitude $\mathrm{h}(\mathrm{t})$, and heading angle $\psi(\mathrm{t})$. The two first variables handle the longitudinal movements of the UAV and the last one the lateral movements.

\section{CHALLENGES AND DIFFICULTIES TO}

\section{ACHIEVE HIGH-PERFORMANCE CONTROL}

The overall flight control objective for the UAV is to track the desired trajectory and the desired heading angle. In addition, the flight control system must maintain the stability of the UAV during all the flight conditions. Full knowledge of the UAV states is required to achieve the control objective, which is in practice not possible.

The most challenge of designing flight control system is to develop a robust nonlinear flight control technique that can sufficiently nullify the UAV dynamics uncertainties and external disturbances especially the wind. There are various sources for uncertainties in aerodynamics for fixed wing UAV. Many of these sources are undefined because the fixed-wing UAV dynamics are natively nonlinear, UAV states are not available or difficult to estimate, and finally, several uncertainties which are unavoidable or imperative in the process [26].

For fixed-wing UAV, the traditional control techniques are not suitable for flight control design because of high nonlinearity and strong coupling between aerodynamic forces and moments. In spite of the dynamic inversion control technique has been utilized for flight control design, its performance is limited in practice because of its design depends on the accurate nonlinear model of the UAV. However, intelligent control techniques have the ability to deal with the inaccurate UAV model [27]

\section{ANFIS}

The neuro-fuzzy inference system proposed by Jang [28] in 1993 is a hybrid intelligent system. ANFIS is a combination between fuzzy logic and artificial neural network in the field of artificial intelligence [29]. Fuzzy logic control includes "If-Then" rules designed by the experts depend on their knowledge and the given database of the plant [30]. It is excellent at dealing with uncertainty and decision making. However, the main defect of fuzzy logic is the inability to learn [31]. On the other hand, the artificial neural network has the ability to learn but don't have the ability to make decisions. The artificial neural network aids in minimizing the error besides to optimize the controller parameters. In ANFIS, the parameters are obtained by trial and error [30]. Harvest the beneficial between two types fuzzy logic and artificial neural network, new hybrid Neuro-Fuzzy systems have come out. In ANFIS, two types of algorithms are employed. The first algorithm is back propagation and the other is hybrid learning algorithm. The hybrid learning algorithm is an aggregation of two algorithms: back propagation algorithm and least mean square algorithm. This combination is employed for training the membership functions of fuzzy logic. The back propagation algorithm is utilized for learning the assumption parameters while the least square algorithm is utilized for learning the consequent parameters [22]. 


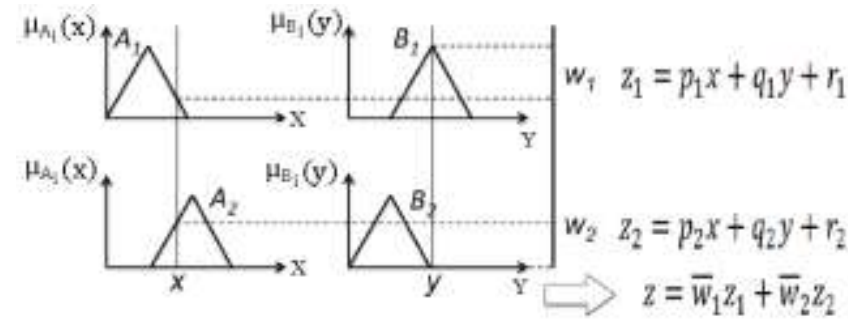

Fig-1: 2-input, 1-output Sugeno fuzzy inference system.

In our proposed approach, the Takagi-Sugeno fuzzy system is assumed to include two inputs $\mathrm{x}$ and $\mathrm{y}$ and one output $\mathrm{z}$. This assumption leads to having two rules. Each rule is a linear combination of its inputs plus constant. The total output will be a weighted average of each rule's output.

Therefore, the Takagi-Sugeno fuzzy system illustrated in Fig-1 will be equivalent to the adaptive neuro-fuzzy system in Fig-2.

Rule 1: if $x$ is $A_{1}$ and $y$ is $B_{1}$ then $z_{1}=p_{1} x+q_{1} y+r_{1}$ Rule 2: if $x$ is $A_{2}$ and $y$ is $B_{2}$ then $z_{2}=p_{2} x+q_{2} y+r_{2}$ where $x$ and $y$ are the two crisp inputs. $p_{i}, q_{i}, r_{i}$ are resulting parameters of the model identified through the training procedure. $A_{i}$ and $B_{i}$ are the fuzzy sets.

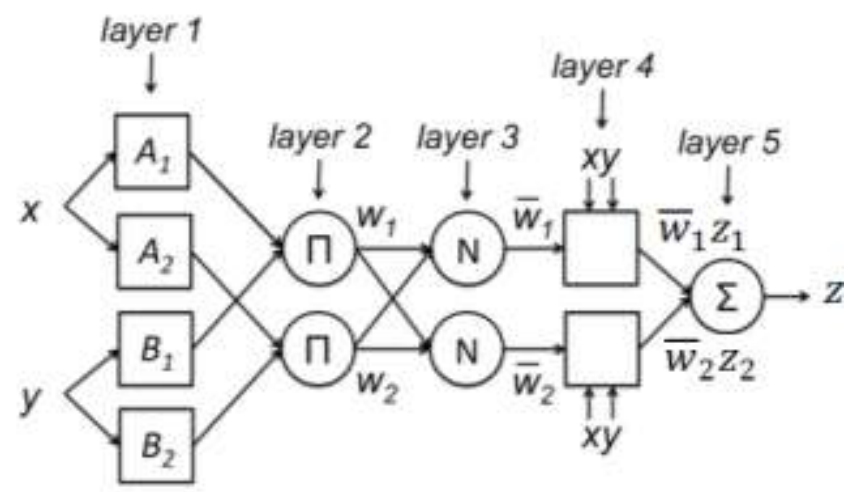

Fig-2: Equivalent ANFIS architecture to Takagi-Sugeno fuzzy inference system.

The structure of ANFIS is shown in Fig-2. It is constructed from 5 layers [32], [33] with the following function:

Layer 1: The first layer is the input node. Each square input node i presents in this first layer has a function such as:

$$
\begin{array}{ll}
\mathrm{O}_{\mathrm{i}}^{1}=\mu_{\mathrm{A}_{\mathrm{i}}}(\mathrm{x}) & \mathrm{i}=1,2 \\
\mathrm{O}_{\mathrm{i}}^{1}=\mu_{\mathrm{B}_{\mathrm{i}}}(\mathrm{y}) & \mathrm{i}=3,4
\end{array}
$$

where $x, y$ are the inputs to the node $i . \mu_{A_{i}}, \mu_{B_{i}}$ are the membership functions of the linguistic variables $A_{i}, B_{i}$ respectively. $\mathrm{O}_{\mathrm{i}}^{1}$ is the membership function which is a triangular as in Fig.1.

Layer 2: The second layer labeled $\Pi$ is the rule nodes. The output of this layer is the product of the two inputs. The minimum value of weights for the two inputs is chosen by this node. The product out is:

$$
\mathrm{w}_{\mathrm{i}}=\mu_{\mathrm{A}_{\mathrm{i}}}(\mathrm{x}) \times \mu_{\mathrm{B}_{\mathrm{i}}}(\mathrm{y}) \quad \mathrm{i}=1,2 .
$$

Layer 3: It is also called average nodes. This layer has circle nodes labeled $\mathrm{N}$ that compute the normalized firing strength as follow:

$$
\overline{\mathrm{w}}_{\mathrm{i}}=\frac{\mathrm{w}_{\mathrm{i}}}{\mathrm{w}_{1}+\mathrm{w}_{2}} \quad \mathrm{i}=1,2
$$

Layer 4: The consequence is produced in this layer. Square nodes in this layer include the linear function of the input signals such as:

$$
\mathrm{O}_{\mathrm{i}}^{4}=\overline{\mathrm{w}}_{\mathrm{i}} \mathrm{z}_{\mathrm{i}}=\overline{\mathrm{w}}_{\mathrm{i}}\left(\mathrm{p}_{\mathrm{i}} \mathrm{x}+\mathrm{q}_{\mathrm{i}} \mathrm{y}+\mathrm{r}_{\mathrm{i}}\right)
$$

where $\left(\mathrm{p}_{\mathrm{i}}, \mathrm{q}_{\mathrm{i}}, \mathrm{r}_{\mathrm{i}}\right)$ are the resulting parameters to be set through the training process. $\overline{\mathrm{w}}_{\mathrm{i}}$ is the normalized firing strength obtaining from layer 3 .

Layer 5: The fifth layer is the final summation layer. The single circle node is labeled $\Sigma$ and sums all the incoming signals coming to this layer.

$$
\mathrm{O}_{\mathrm{i}}^{5}=\sum_{\mathrm{i}} \overline{\mathrm{w}}_{\mathrm{i}} \mathrm{z}_{\mathrm{i}}
$$

Now, the ANFIS model is trained to produce the desired controller performance by adapting the membership functions and the ANFIS parameters. Membership functions can be chosen depend on experience. However, in the case of unavailability of the expert, the membership functions are chosen by trial and error [33].

\section{SIMULATION AND RESULTS}

In this work, MATLAB ANFIS Editor Toolbox is utilized to design the inference engines. MATLAB and Aerosim software are utilized to study the performance of the designed flight control system.

\subsection{Longitudinal Autopilot}

For longitudinal autopilot, the inference system for altitude control has 7 membership functions and 49 fuzzy rules as shown in Fig-3. However, the inference system for speed control has 5 membership functions and 25 rules. Two inputs are considered for the inference system: error and change of error. The input membership functions for altitude control are shown in Fig-4 and Fig-5. The output surface for altitude controller is shown in Fig-6. The input membership functions for speed control are shown in Fig-7 and Fig-8. The output surface for speed controller is shown in Fig-9. 


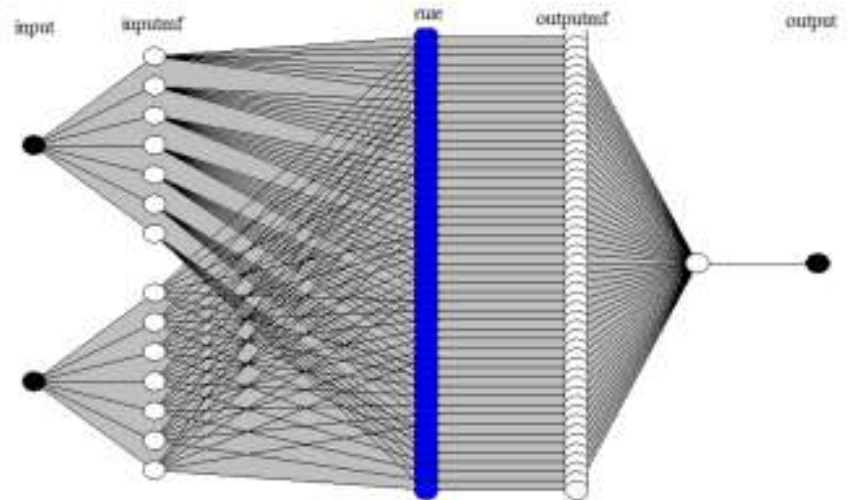

Fig-3: ANFIS architecture of fuzzy controller model for altitude control.

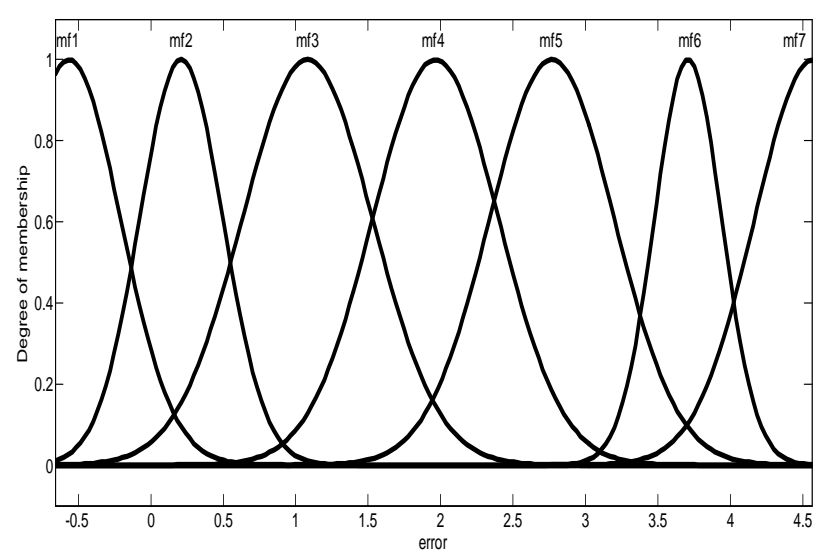

Fig-4: Input variable "error" for altitude control.

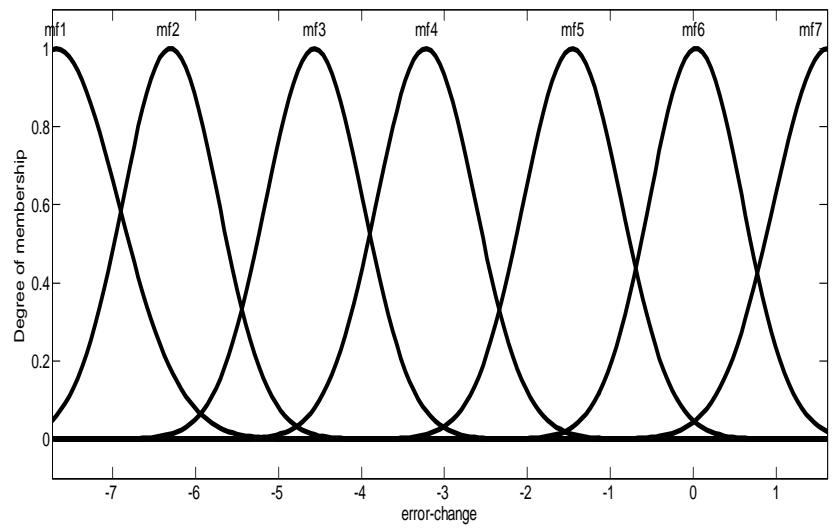

Fig-5: Input variable "error_change" for altitude control.

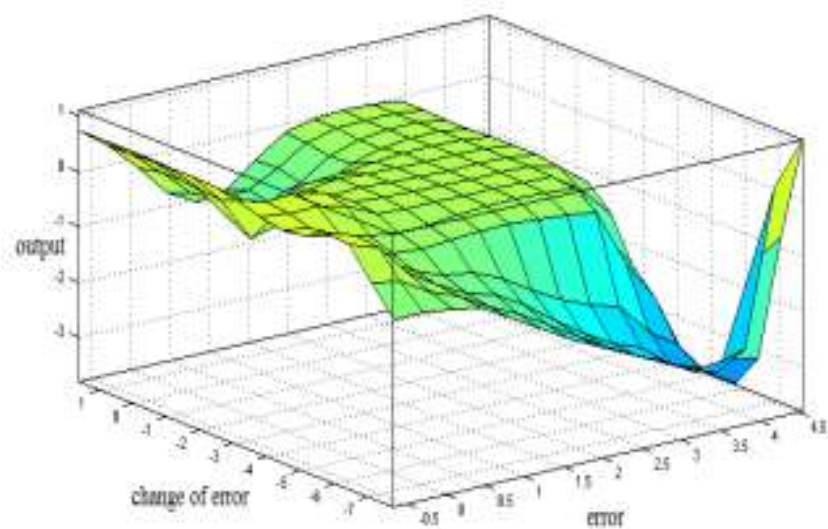

Fig-6: Output surface of fuzzy modules for altitude control.

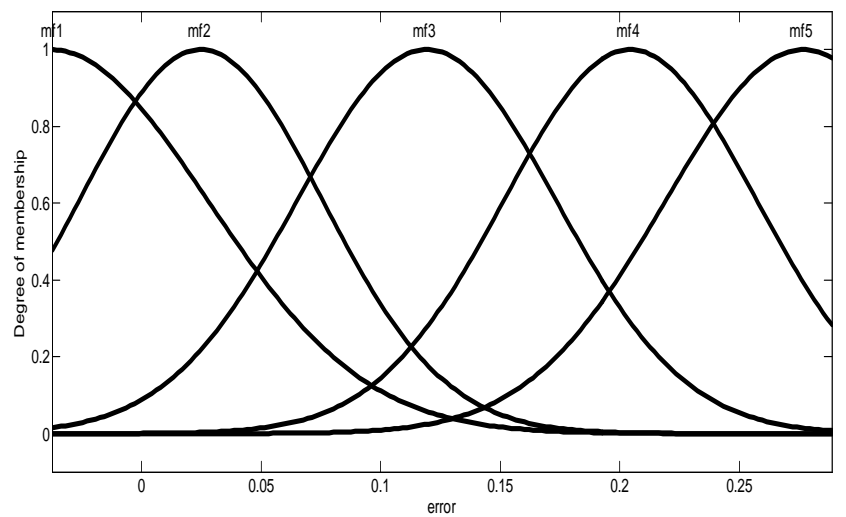

Fig-7: Input variable "error" for speed control.

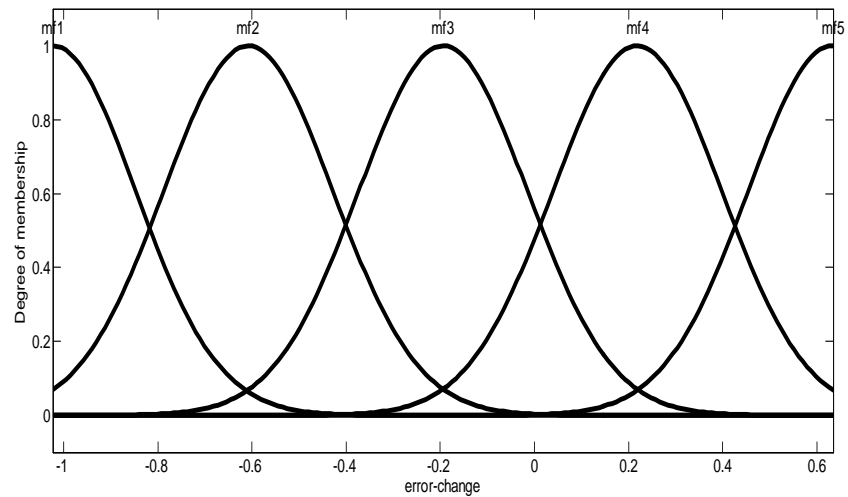

Fig-8: Input variable "error_change" for speed control.

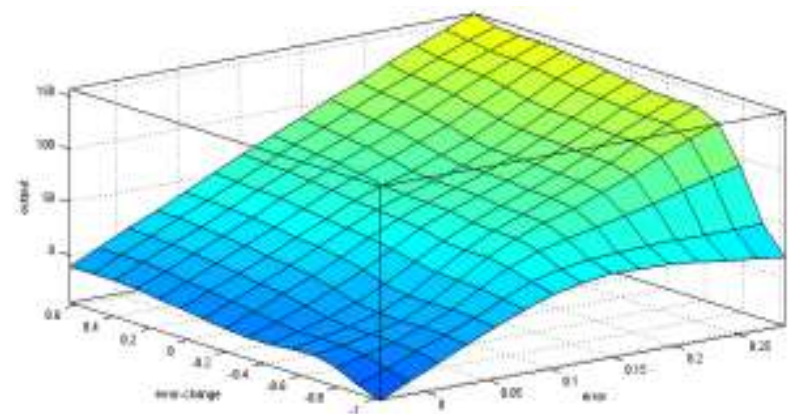

Fig-9: Output surface of fuzzy modules for speed control.

The output response for altitude control of longitudinal autopilot is shown in Fig. 10.

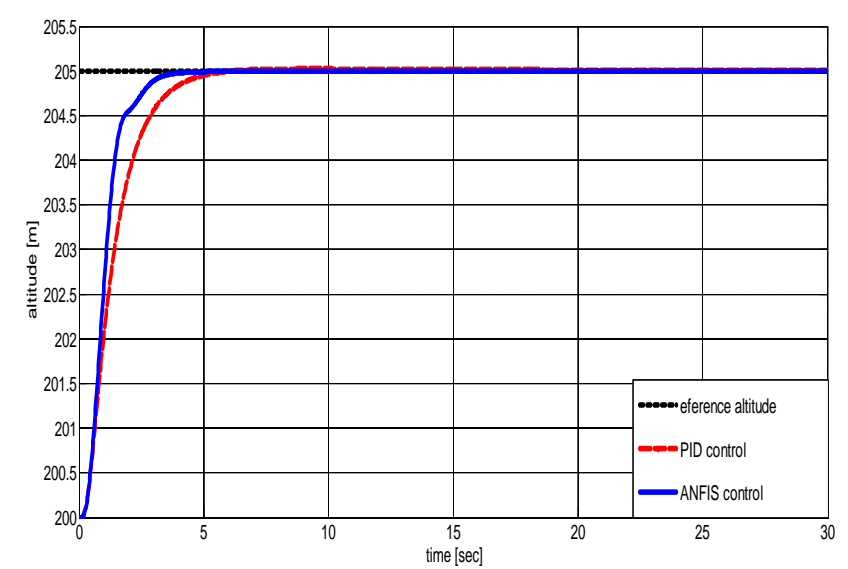

Fig-10: Step response of altitude control. 
The output response for speed control of longitudinal autopilot is shown in Fig-11.

The proposed altitude controller based on ANFIS is tested to track different altitude values. The response of the altitude control for tracking different altitudes is shown in Fig-12.

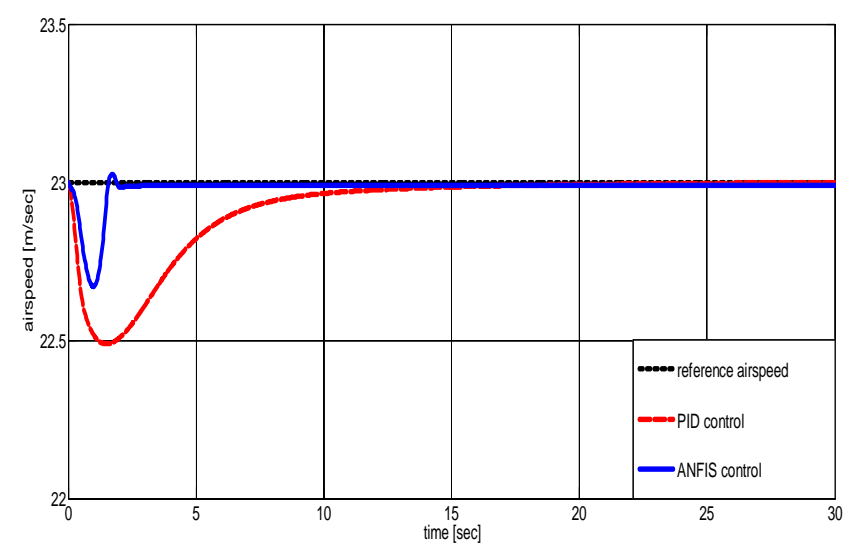

Fig-11: Speed-hold longitudinal autopilot response.

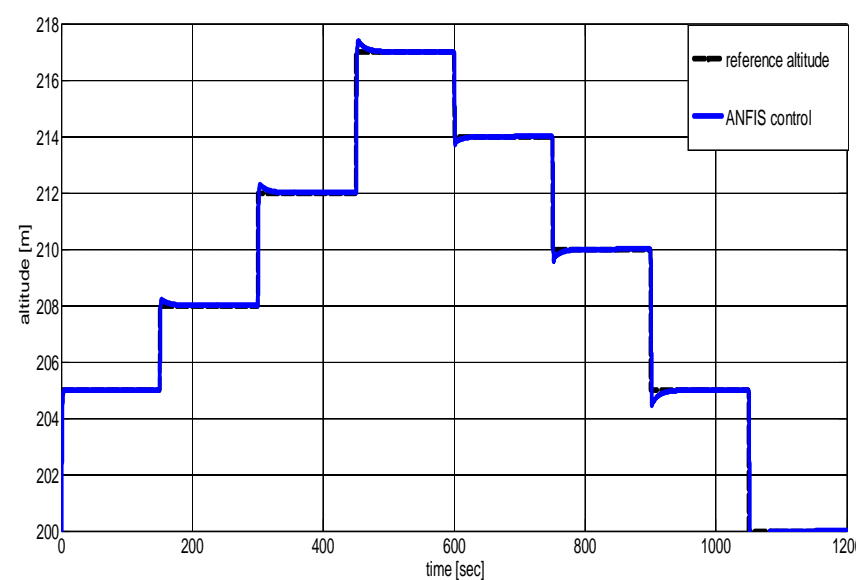

Fig-12: ANFIS altitude controller for tracking different altitudes.

The proposed speed controller based on ANFIS is tested to track different speed values. The response of the altitude control for tracking different airspeeds is shown in Fig-13.

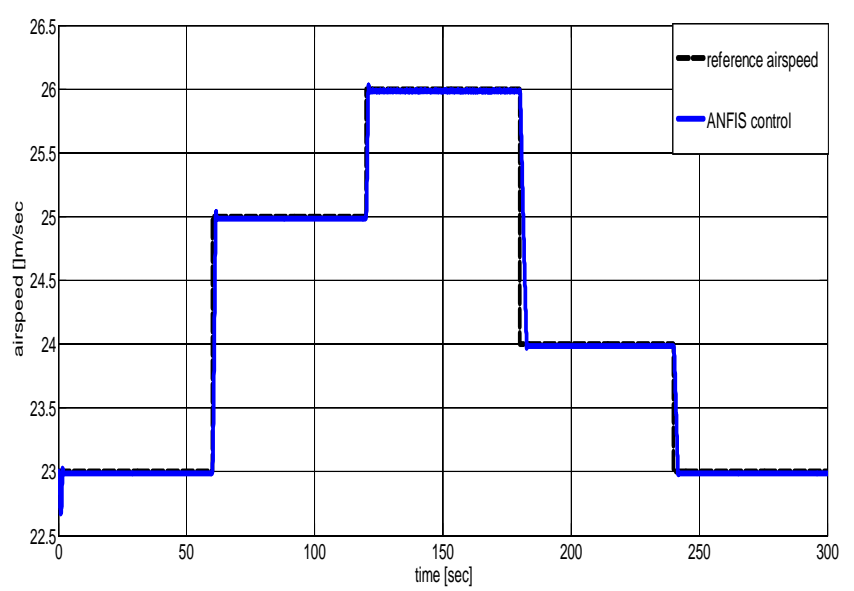

Fig-13: ANFIS speed controller for tracking different airspeeds.

\subsection{Lateral Autopilot}

For lateral autopilot, the inference system for heading angle control has 5 membership functions and 25 rules. The inputs membership functions for heading control are shown in Fig14 and Fig-15.

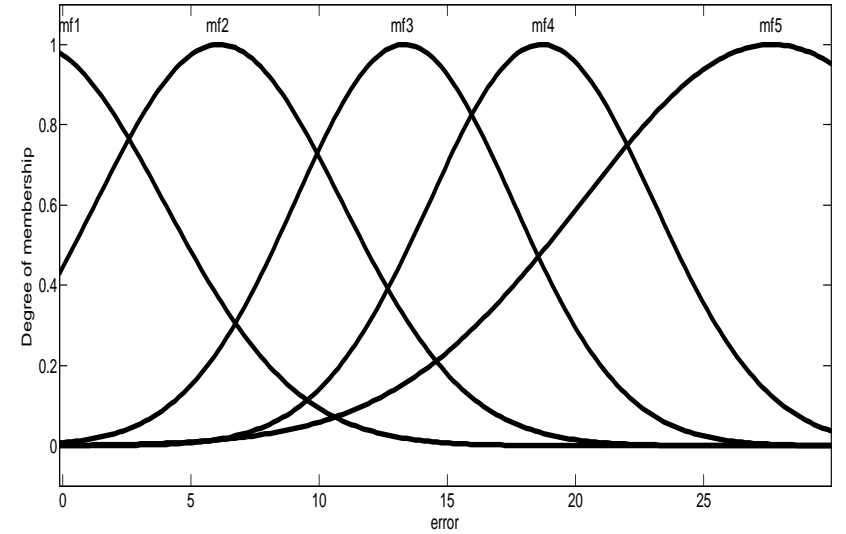

Fig-14: Input variable "error" for heading control.

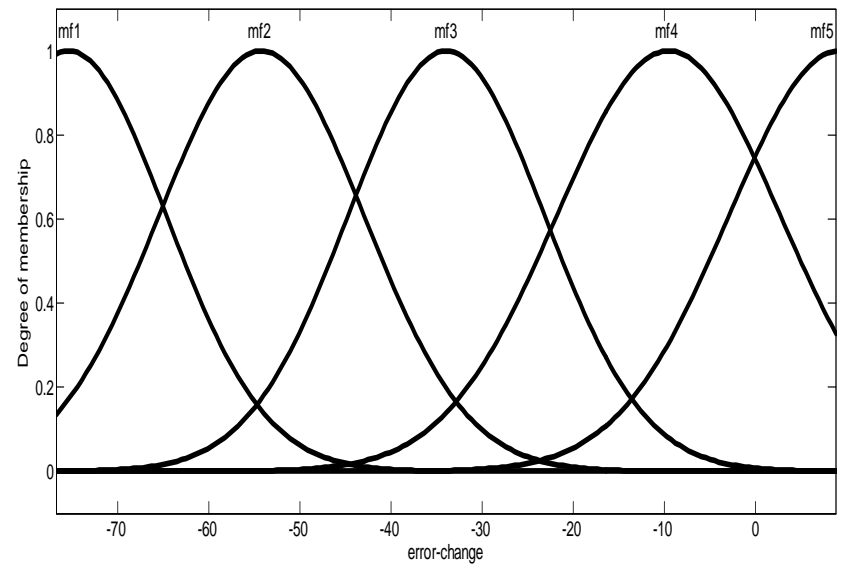

Fig-15: Input variable "error_change" for heading control.

The output surface of fuzzy modules for heading control is shown in Fig. 16.

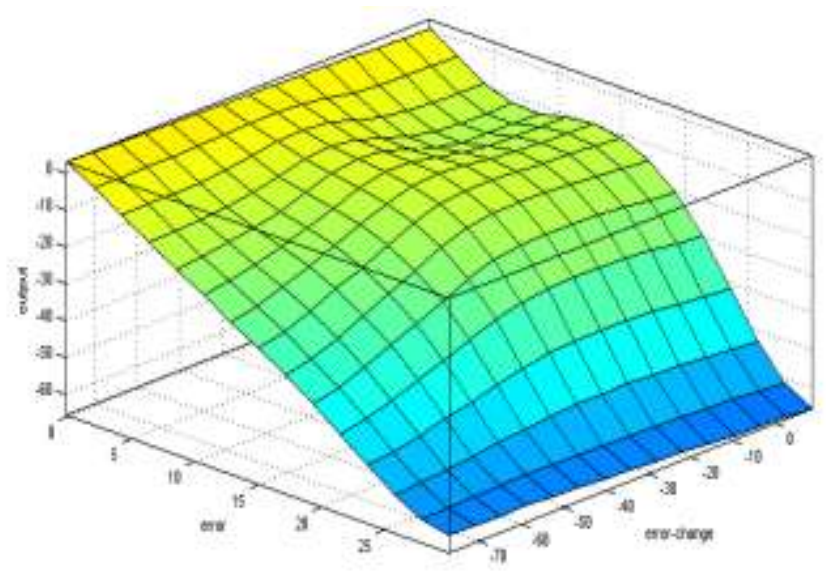

Fig-16: Output surface of fuzzy modules for heading control.

The output response for heading control of lateral autopilot is shown in Fig-17. 
The proposed heading controller based on ANFIS is tested to track different heading angles values. The response of the heading control for tracking different heading angles is shown in Fig-18.

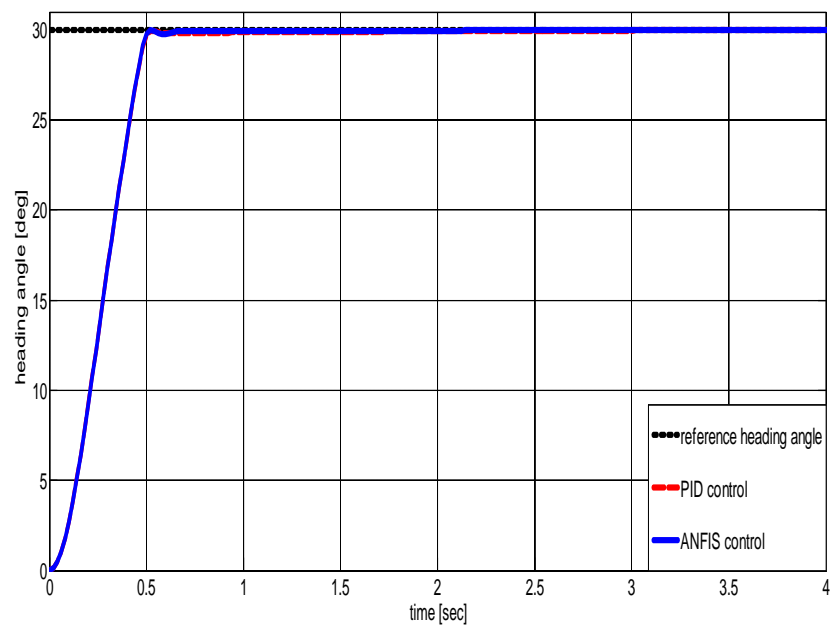

Fig-17: Step response of heading control.

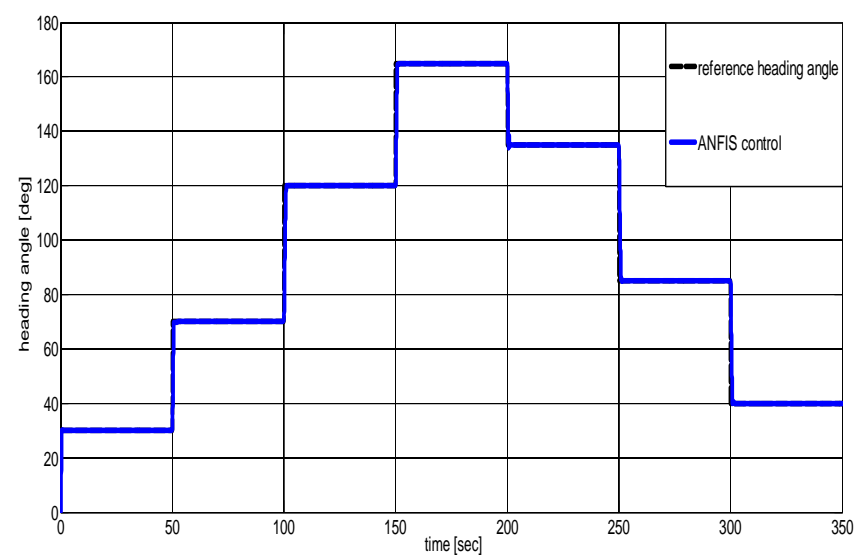

Fig-18: Lateral ANFIS autopilot response for tracking different heading angles.

Finally, the proposed autonomous flight controller is tested to track reference flight path. The response of the proposed controller is shown in Fig-19.

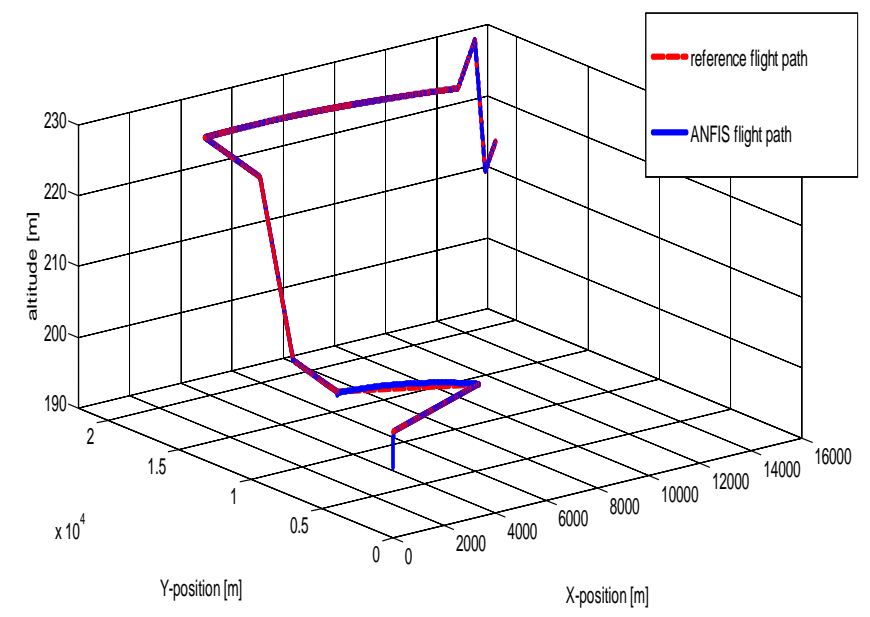

Fig-19: ANFIS based autonomous flight controller response.

\subsection{ANFIS Performance against Parametric}

\section{Uncertainties}

The robustness of the proposed flight controller in the presence of UAV parametric uncertainties is validated and tested in this subsection. In our design, stability derivatives, weight, moments of inertia, Mach no., dynamic pressure, and c.g. are selected for parametric uncertainty analysis as in Table-1.

Table -1: UAV parameters with their uncertainties

\begin{tabular}{|c|l|l|l|}
\hline Parameter & $\begin{array}{l}\text { Prediction } \\
\text { uncertainty(\%) }\end{array}$ & Parameter & $\begin{array}{l}\text { Prediction } \\
\text { uncertainty(\%) }\end{array}$ \\
\hline $\mathrm{C}_{\mathrm{L}}$ & 60 & $\mathrm{P}_{\mathrm{dyn}}$ & 50 \\
\hline $\mathrm{C}_{\mathrm{D}}$ & 60 & Mach no. & 40 \\
\hline $\mathrm{C}_{\mathrm{Y}}$ & 60 & weight & 60 \\
\hline $\mathrm{C}_{\mathrm{m}}$ & 60 & c.g. & 50 \\
\hline $\mathrm{C}_{\mathrm{l}}$ & 60 & $\mathrm{~J}_{\mathrm{x}}, \mathrm{J}_{\mathrm{y}}, \mathrm{J}_{\mathrm{z}}, \mathrm{J}_{\mathrm{xz}}$ & 70 \\
\hline $\mathrm{C}_{\mathrm{n}}$ & 60 & & \\
\hline
\end{tabular}

Robustness of the flight control system is illustrated by varying all the chosen variables to their maximum uncertainties during the simulation are running to obtain the maximum singular value. Fig-20, Fig-21, and Fig-22 demonstrate the robust stability and the robust performance of the designed flight control system.

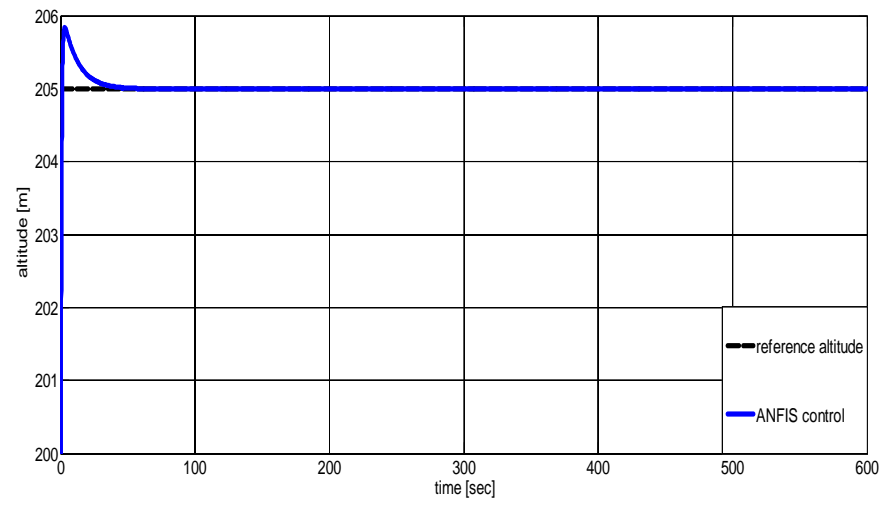

Fig-20: Step response of altitude control with parametric uncertainties.

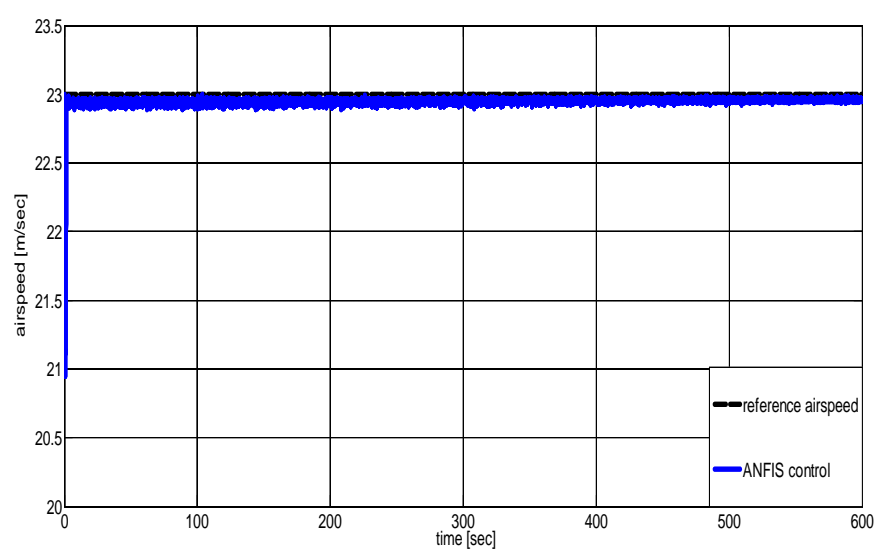

Fig-21: Step response of speed control with parametric uncertainties. 


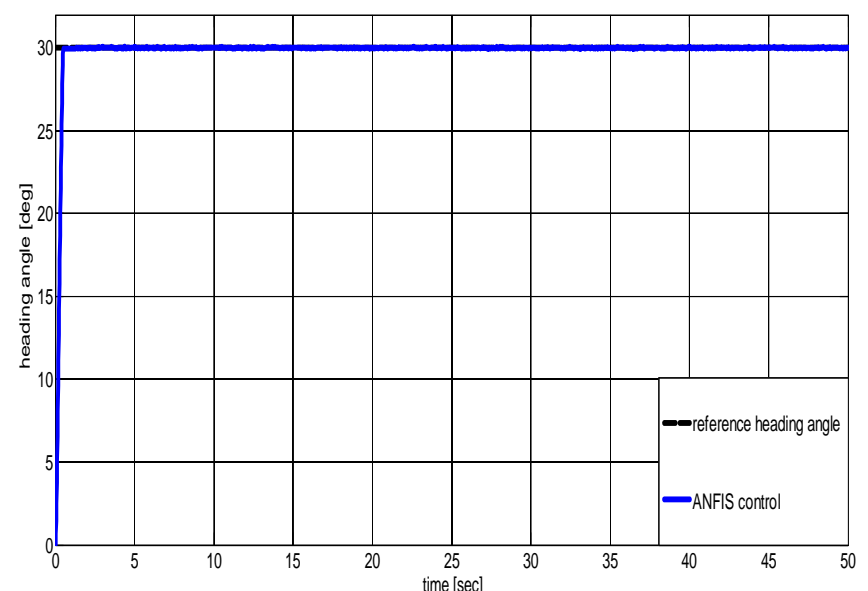

Fig-22: Step response of heading control with parametric uncertainties.

\subsection{ANFIS Performance against Disturbance}

\section{Rejection}

The effect of crosswind disturbance in the $\mathrm{X}-\mathrm{Y}$ plane is studied in this subsection. The UAV is subjected to crosswind disturbance in the $X-Y$ plane with value $5 \mathrm{~m} / \mathrm{s}$ in each direction. The robustness of the flight control system is shown if Fig-23, Fig-24, and Fig-25.

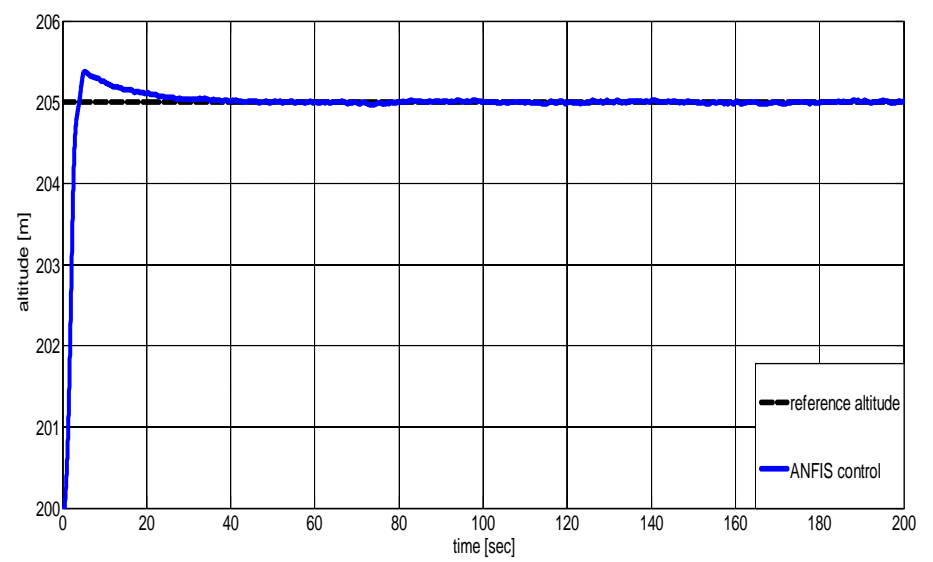

Fig-23: Step response of altitude control with wind disturbance.

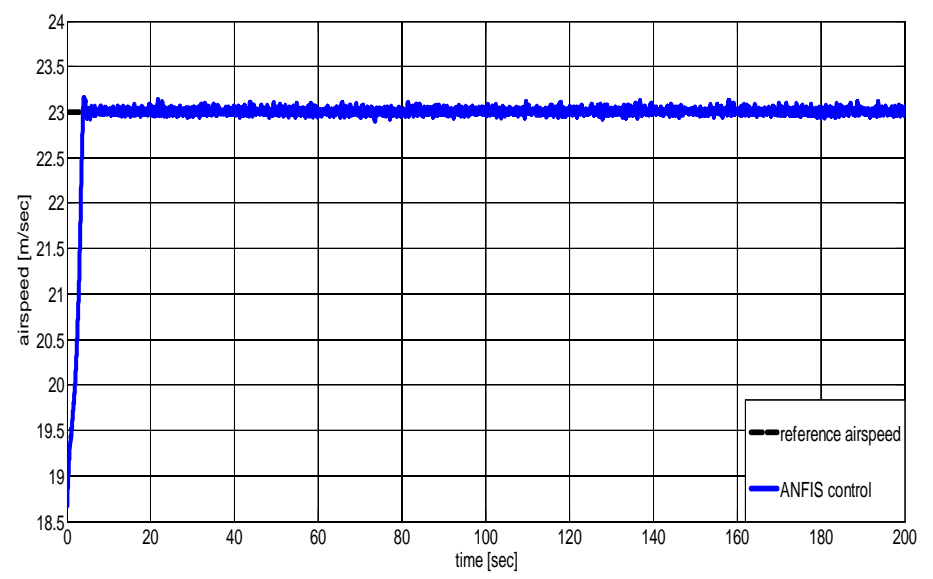

Fig-24: Step response of airspeed control with wind disturbance.

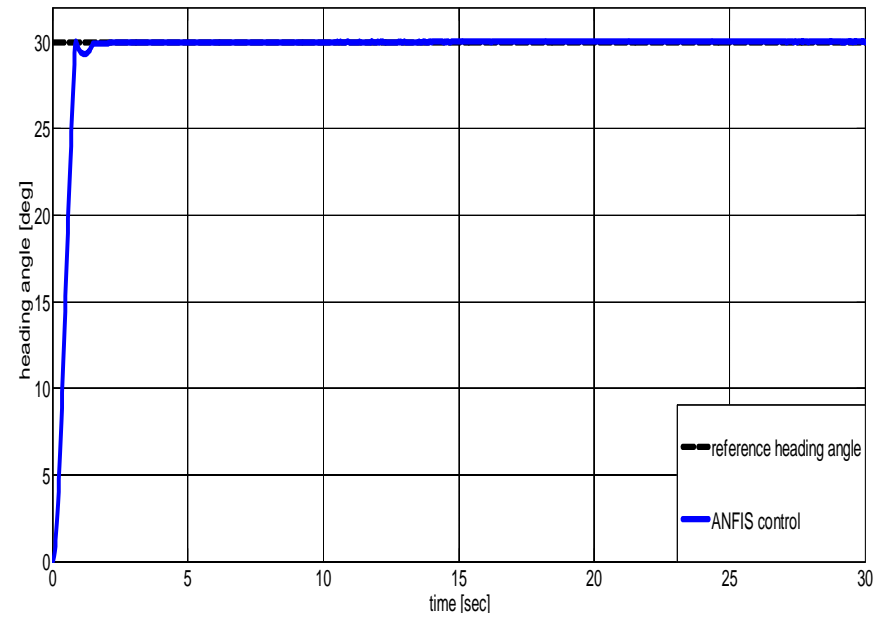

Fig-25: Step response of heading control with wind disturbance.

\section{CONCLUSION}

The main objective of the autonomous flight controller is to allow the UAVs to perform their task autonomously, without any input from the ground operator. The Adaptive Neuro-Fuzzy Inference System described in this paper can be employed to endue a capacity of autonomy to fixed-wing UAV. A series of simulation results demonstrate the feasibility and advantages of the designed autonomous flight control of UAVs. The proposed flight control system produces performance better than traditional PID controller. The properties responses in tracking a reference set point are enhanced. The simulations studies demonstrate that the proposed ANFIS flight controller of UAV can track the desired trajectories successfully even in UAV parametric uncertainties and wind disturbances.

\section{ACKNOWLEDGEMENT}

This work was partly supported by National Natural Science Foundation of China (Grant Nos. 61273350 and U1435220), Beijing Science and Technology Project of China (Grant No. D16110400130000-D161100001316001).

\section{REFERENCES}

[1] Syed Ussama Ali, M. Zamurad Shah and Raza Samar, "Sliding Mode Based Roll Control Law Design for a Research UAV and its Flight Validation", IEEE 13th International Bhurban Conference on Applied Sciences and Technology (IBCAST), Pakistan, 2016.

[2] Sefer Kurnaz, Omer Cetin, and Okyay Kaynak, "Adaptive neuro-fuzzy inference system based autonomous flight control of unmanned air vehicles", Elsevier Expert Systems with Applications, 2010.

[3] A.M. Klipa and A.D. Sydorenko, "On One Approach to Small UAV Flight Control System Design based on Reduced-Order Observer", IEEE $3^{\text {rd }}$ International Conference Actual Problems of Unmanned Aerial Vehicles Developments (APUAVD), Kiev, 2015

[4] Abdel Moneim, Idris Moutaman Mirghani, Abdel Rahim Satti and Ahmed A. Imam, "Development of Computer Aided Tools for UAV Flight Control", IEEE 
International Conference on Computing, Electrical, and Electronics Engineering (ICCEEE), Khartoum, 2013.

[5] Shengyi Yang, Kunqin Li, and Jiao Shi, "Design and Simulation of the Longitudinal Autopilot of UAV Based on Self-Adaptive Fuzzy PID Control," IEEE International Conference on Computational Intelligence and Security, vol. 1, Beijing, 2009.

[6] W.T.M.S Tennakoon and S.R. Munasinghe, "Design and simulation of a UAV controller system with high maneuverability," 4th IEEE International Conference on Information and Automation for Sustainability ICIAFS, Colombo, 2008.

[7] Liwei Qiu, Jianqiang Yi, Guoliang Fan, Wensheng Yu and Ruyi Yuan, "Design of Robust Backstepping Controller for Unmanned Aerial Vehicle Using Analytical Redundancy and Extended State Observer", 3rd IEEE International Symposium on Systems and Control in Aeronautics and Astronautics (ISSCAA), Harbin, 2010.

[8] S. Park, J. Deystt, and J. P. How, "Performance and Lyapunov stability of a nonlinear path-following guidance method," J. Guidance, Control, Dyn., vol. 30, no. 6, pp. 1718-1728, 2007.

[9] Ji-hong and Zhu, "A Survey of Advanced Flight Control Theory and Application," IEEE IMACS Multiconference on Computational Engineering in Systems Applications (CESA), China, 2006.

[10] Liwei Qiu, Guoliang Fan, Jianqiang Yi, and Wensheng Yu," Design of Neural Network and Backstepping based Adaptive Flight Controller for Multi-Effector UAV", IEEE International Conference on Robotics and Biomimetics, 2009.

[11] Liwei Qiu, Guoliang Fan, Jianqiang Yi, and Wensheng $\mathrm{Yu}$, "Robust Hybrid Controller Design based on Feedback Linearization and $\mu$ Synthesis for UAV", $2^{\text {nd }}$ IEEE International Conference on Intelligent Computation Technology and Automation, vol. 1, Hunan 2009.

[12] Puneet Wahi, Ravi Rania and Fahmida N Chowdhury, "A Survey of Recent Work in Adaptive Flight Control", Proceedings of the $33^{\text {rd }}$ IEEE Southeastern Symposium on System Theory, Athens, 2001.

[13] P.B. Sujit and Srikanth Saripalli, and João Borges Sousa, "A survey and analysis of algorithms for fixedwing unmanned aerial vehicles," IEEE CONTROL SYSTEMS MAGAZINE, vol. 34, pp. 42 - 59, 2014.

[14] R. Aruneshwaran, S. Suresh, J. Wang, T.K. Venugopalan, Neural adaptive flight controller for ducted-fan UAV performing nonlinear maneuver, IEEE Symposium on Computational Intelligence for Security and Defense Applications (CISDA), pp. 51-56, 2013.

[15] Hongda Chen, Kuochu Chang, C.S. Agate, UAV Path Planning with Tangent-plus-Lyapunov Vector Field Guidance and Obstacle Avoidance, IEEE Transactions on Aerospace and Electronic Systems, Vol. 49, Issue 2, pp. 840-856, 2013.

[16] Zhufeng Xie, Yuanqing Xia, Mengyin Fu, Robust trajectory-tracking method for UAV using nonlinear dynamic inversion, IEEE 5th International Conference on Cybernetics and Intelligent Systems (CIS), pp. 9398, 2011.
[17] Mario Collotta, Giovanni Pau, and Riccardo Caponetto, "A Real-Time System based on a Neural Network Model to Control Hexacopter Trajectories", IEEE International Symposium on Power Electronics, Electrical Drives, Automation and Motion (SPEEDAM), Ischia, 2014.

[18] Pourya Shahmaleki and Mojtaba Mahzoon, " GA modeling and ANFIS control design for a solar power plant", IEEE, American Control Conference (ACC), 2010.

[19] K. M. Udofia and J. O. Emagbetere, "Development of multi-agent ANFIS-based model for urban traffic signal control", IEEE International Conference on Connected Vehicles and Expo (ICCVE), Las Vegas,2013.

[20] Xiong Luo, Yi Chen, Jennie Si, and Feng Liu, "Longitudinal control of hypersonic vehicles based on direct heuristic dynamic programming using ANFIS", IEEE, International Conference on Neural Networks (IJCNN), 2014

[21] Chun-Fei Hsu, Chih-Min Lin, and Rong-Guan Yeh, "Supervisory adaptive dynamic RBF-based neuralfuzzy control system design for unknown nonlinear systems", Elsevier, Applied Soft Computing, Volume 13, Issue 4, April 2013, Pages 1620-1626.

[22] Xiujia Chen and Xianku Zhang, "Nonlinear Feedback Control Based on ANFIS", 12th IEEE International Conference on Fuzzy Systems and Knowledge Discovery (FSKD), Zhangjiajie, 2015 .

[23] Stevens B.L. and F.L. Lewis. "Aircraft Control and Simulation." John Wiley \& Sons, Inc. second edition, 2003.

[24] Rafael Socas, Sebastian Dormido and Raquel Dormido, "Event-based control strategy for the guidance of the Aerosonde UAV," IEEE European Conference on Mobile Robots (ECMR), pages 1-6, 2015.

[25] Beard, R.W., and McLain, T.W., "Small unmanned aircraft. Theory and practice" Princeton University Press, 2012.

[26] Nickolas Grady, Michael Frye, and Chunjiang Qian, "The Development of a High Performance UAV for Flight Control Research", Region 5 Conference IEEE, 2006.

[27] Xinmin Wang, Rong Xie, and Yan Li, "Nonlinear Controller Design for a super maneuverable Aircraft", IEEE International Joint Conference on Computational Sciences and Optimization, 2009.

[28] J.S.R. Jang, "ANFIS: Adaptive Network-Based Fuzzy Inference System", IEEE Transactions on Systems, Man, and Cybernetics, 1993.

[29] Samarjit Kar, Sujit Das, and Pijush Kanti Ghosh, "Applications of neuro fuzzy systems: A brief review and future outline", Elsevier Applied Soft Computing, 2014.

[30] Nandkishor Joshi and Bhavana Jharia, "Optimized fuzzy power control over fading channels in spectrum sharing cognitive radio using ANFIS", $2^{\text {nd }}$ IEEE International Conference on Signal Processing and Integrated Networks (SPIN), Noida, 2015.

[31] John Darvill, Alin Tisan, and Marcian Cirstea, "An ANFIS-PI Based Boost Converter Control Scheme", 
$13^{\text {th }}$ IEEE International Conference on Industrial Informatics (INDIN), Cambridge, 2015.

[32] Rinu Simon and A. Geetha, "Comparison on the Performance of Induction Motor Control Using Fuzzy and ANFIS Controllers", IEEE International Conference on Emerging Trends in Computing, Communication and Nanotechnology (ICE-CCN), Tirunelveli, 2013.

[33] L. A. Torres-Salomao and J. Anzurez-Marin, "Adaptive Neuro-Fuzzy Inference System Control for a Two Tanks Hydraulic System Model", Power, IEEE International Autumn Meeting on Electronics and Computing (ROPEC), Mexico City, 2013.

\section{BIOGRAPHIES}

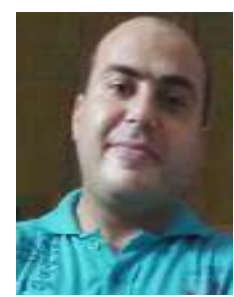

Amr Sarhan received the B.S. degree in electrical engineering from Military Technical College, Cairo, Egypt, in 2002; senior researcher until 2006. In 2011 he has received the M.S. degree from Military Technical College, Cairo, Egypt; lecture assistant in aircraft special equipment department at Military Technical College, Cairo, Egypt. He is interested in unmanned aerial vehicles flight control systems. He currently a Ph.D. candidate in UAV flight control system from School of Automation Science and Electrical Engineering in Beihang University (Beijing University of Aeronautics and Astronautics), Beijing, China.

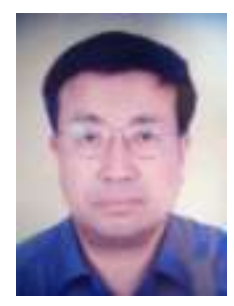

Shiyin Qin received his Bachelor's and Master's degrees in Engineering Science in Automatic Controls and Industrial Systems Engineering from Lanzhou Jiaotong University in 1978 and 1984, respectively, and his Ph.D. degree in Industrial Control Engineering and Intelligent Automation from Zhejiang University in 1990. He is now a Professor at the School of Automation Science and Electrical Engineering in Beihang University (Beijing University of Aeronautics and Astronautics). His current major research topics include image processing and pattern recognition, intelligent control systems of UAVs, intelligent optimizing controls of large scale multi-robot hybrid systems, complex systems and complexity science, etc. 